\title{
TESTE DE RESISTÊNCIA DE FORÇA DE MEMBROS SUPERIORES PARA IDOSOS: COMPARAÇÃO ENTRE HALTERES COM PESOS DIFERENTES
}

\author{
STRENGTH RESISTANCE TEST OF SUPERIOR MEMBERS FOR ELDERLY \\ PEOPLE: COMPARISON BETWEEN DUMBBELLS WITH DIFFERENT WEIGHTS
}

\begin{abstract}
RESUMO
O objetivo do estudo foi comparar o desempenho de idosos no teste de resistência de força de membros superiores (RESISFOR) com halteres de diferentes pesos (1,8 kg e $2 \mathrm{~kg}$ para mulheres e 3,6 kg e $4 \mathrm{~kg}$ para homens). A amostra foi constituída por 407 idosos (349 feminino e 58 masculino), com idade de 60 a 88 anos, praticantes de exercício físico por, no mínimo, seis meses. O instrumento utilizado foi o RESISFOR da bateria de testes American Alliance for Health, Physical Education, Recreation \& Dance (AAHPERD). Foram realizadas duas execuções do teste com halteres de pesos diferentes, RESISFOR (1,8 Kg e 3,6 Kg) e RESISFOR “ajustado" (2 Kg e $4 \mathrm{Kg}$ ). Para a análise dos dados, procedeu-se a análise descritiva, Teste $t$ de Student para amostras independentes, Teste $t$ pareado para amostras dependentes e ANOVA com post-hoc de Tukey, adotando-se nível de significância de $5 \%$. Os resultados demonstraram que a utilização de halteres com pesos de 2 e 4 kg, parece não afetar o desempenho dos idosos, principalmente do sexo masculino. Para o sexo feminino, detectou-se diferença significativa nas médias na segunda comparação realizada para o gênero. Todavia, essa diferença pode ter sido decorrente de fatores externos ao teste, visto que, as maiores médias obtidas foram para o RESISFOR "ajustado". Diante disso, busca-se facilitar ainda mais a avaliação da capacidade funcional da população idosa, sendo recomendada, para o RESISFOR, a utilização de halteres com peso de $2 \mathrm{Kg}$ para mulheres e 4 kg para os homens, que são de baixo custo, fácil aquisição e acesso no comércio brasileiro.
\end{abstract}

Palavras-chave: Idoso; Resistência de força; Avaliação; Testes de aptidão; Atividade física.

\section{ABSTRACT} The aim of the study was to compare the performance of elderly people in the strength resistance test of
superior members (SRTSM) with dumbbells of different weights (1,8 kg and $2 \mathrm{~kg}$ for women and $3,6 \mathrm{~kg}$ and $4 \mathrm{~kg}$ for men). The sample consisted of 407 elderly (349 feminine and 58 masculine), with age of 60 to 88 years, practitioners of physical exercise for at least six months. The used instrument was the SRTSM of the battery of tests American Alliance for Health, Physical Education, Recreation and Dance (AAHPERD). Two executions of the test were carried through with dumbbells of different weights, SRTSM (1,8 kg and 3,6 kg) and "adjusted" SRTSM (2 kg and $4 \mathrm{~kg})$. For the analysis of the data, it was proceeded descriptive analysis, test $t$ of Student for independent samples, paired test for dependent samples and ANOVA with post-hoc of Tukey, adopting itself the $5 \%$ level significance. The results demonstrated that the use of dumbbells with 4 weights of 2 and $\mathrm{kg}$, seem not to affect the performance of the elderly ones, mainly of the masculine sex. For the feminine sex significant difference in the averages of the second comparison carried through for the sort was detected. However, this difference can have been resulting from external factors to the test, since, the greater average obtained was for "the adjusted" SRTSM. Nevertheless, one tries to facilitate even more the evaluation of the functional capacity of the elderly population, being recommended for SRTSM the use of dumbbells with weight of $2 \mathrm{~kg}$ for women and $4 \mathrm{~kg}$ for men that they are of low cost, easy acquisition and access in the Brazilian market.

Key words: Elderly; Strength resistance; Evaluation; Aptitude Tests; Motor activity.

1 Programa de Pós-Graduação em Educação Física da Universidade Federal de Santa Catarina; Bolsista CAPES. 2 Programa de Pós-Graduação em Ciências do Movimento Humano da Universidade do Estado de Santa Catarina - UDESC. 3 Universidade Federal de Santa Catarina, Centro de Desportos, CDS/UFSC. Brasil. 4 Laboratório de Gerontologia. Centro de Ciências da Saúde e do Esporte. Universidade do Estado de Santa Catarina. LAGER/CEFID/UDESC. Brasil.

5 Departamento de Informática e Estatística. Universidade Federal de Santa Catarina. 


\section{INTRODUÇÃO}

O fenômeno do envelhecimento populacional vem ganhando evidência em todo o mundo, principalmente nos países em desenvolvimento, nos quais este processo ocorre de forma acelerada ${ }^{1}$. No Brasil, estima-se que por volta de 2020 haverá cerca de 30 milhões de idosos. Não apenas o número de pessoas idosas vem aumentando, mas também a expectativa de vida da população. A expectativa média de vida do brasileiro, ao nascer, passou de 66,93 anos em 1991, para 72,05 anos em $2005^{2}$. Segundo dados do censo demográfico de $2000^{3}$,o brasileiro vive apenas 54 anos livre de incapacidades funcionalmente limitantes, o que de acordo com a expectativa de vida no ano de 2005 resultaria em 18,05 anos com algum tipo de limitação ou dependência.

Nesse sentido, o nível de autonomia e a preservação da independência do idoso relacionamse com a manutenção da capacidade funcional e com a promoção de saúde. Diante disso, a avaliação da mesma assume relativa importância para que se criem estratégias, visando melhorias tanto para a saúde como para a qualidade de vida dos idosos.

Em um estudo de revisão acerca dos instrumentos de avaliação da capacidade funcional em idosos ${ }^{4}$, os autores identificaram que os métodos habituais consistem na observação direta (testes de desempenho) e questionários, quer auto-aplicados ou concebidos por entrevistas face a face. Na área da saúde, especificamente, na Educação Física, tem-se utilizado diversas baterias de testes motores, dentre essas, a Continuous-scale physical functional performance (CS-PFP), a American Alliance for Health, Physical Education, Recreation \& Dance (AAHPERD) e a Fullerton Tests ${ }^{5}$.

Um dos instrumentos que merece destaque é a bateria AAHPERD, a qual é composta por cinco testes motores (coordenação, resistência de força de membros superiores, flexibilidade, agilidade e equilíbrio dinâmico, resistência aeróbia geral $)^{6}$. Esta bateria atende critérios de validade e confiabilidade ${ }^{5}$ e, com objetivo de facilitar a avaliação e comparação dos inúmeros grupos de idosos nas várias regiões do Brasil, valores normativos para a população brasileira já foram estabelecidos para algumas faixas etárias ${ }^{7,8} \mathrm{e}$ continuam sendo desenvolvidos para as demais ${ }^{5}$.

Contudo, em se tratando de uma bateria norteamericana, em determinados testes a tradução das unidades de medida para a realidade brasileira, acaba gerando valores fracionados, como no caso específico deste estudo, o teste de resistência de força de membros superiores (RESISFOR). Neste teste, a bateria da AAHPERD propõe o uso de um halter de 8 libras para os homens e 4 libras para as mulheres, sendo que, na conversão para quilos este valor é dado por 1,8 kg (mulheres) e 3,6 kg (homens). Observa-se, no comércio brasileiro, dificuldade de encontrar halteres com este peso, o que requer desgaste de halteres de $2 \mathrm{Kg}$ e $4 \mathrm{~kg}$ (valores mais próximos encontrados no mercado nacional), para que fiquem com o peso recomendado. Para o desgaste dos halteres, é necessário equipamento mecânico, balança de alta precisão e custo financeiro, o que pode dificultar a aquisição desse material.

Assim, a aplicação do RESISFOR com halteres de $2 \mathrm{~kg}$ para mulheres e $4 \mathrm{~kg}$ para homens, sem implicações na sua validade, favorecerá a aquisição no mercado brasileiro, bem como, contribuirá para avaliação: da resistência de força dos membros superiores, do índice de aptidão funcional geral (resultado do somatório dos cinco testes da bateria da AAHPERD) e da capacidade funcional, contribuindo para a realização de novos estudos.

Diante disto, verifica-se a necessidade de analisar o desempenho dos idosos no RESISFOR, utilizando halteres com medidas exatas. Portanto, este estudo objetivou comparar o desempenho de idosos no teste de resistência de força de membros superiores com halteres de diferentes pesos (1,8 kg e $2 \mathrm{~kg}$ para mulheres) e (3,6 kg e 4 kg para homens).

\section{PROCEDIMENTOS METODOLÓGICOS}

Este estudo obteve aprovação do Comitê de Ética para Seres Humanos da Universidade Federal de Santa Catarina (UFSC), processo $n^{\circ}$. 050/05. Todos os idosos foram informados sobre o objetivo da pesquisa e assinaram o termo de consentimento livre e esclarecido - TCLE.

\section{População e Amostra}

A população deste estudo foi de 839 idosos, participantes do Programa de Ginástica e Atividades Aquáticas para a Terceira Idade - UFSC e Grupo de Estudos da Terceira Idade da Universidade do Estado de Santa Catarina - GETI/UDESC.

Os critérios de seleção da amostra foram: idosos com idade igual ou superior a 60 anos; praticante de exercício físico por, no mínimo, seis meses; ter realizado o RESISFOR com halteres de pesos diferentes na mesma avaliação (duas vezes).

A amostra foi constituída por 407 idosos (349 mulheres e 58 homens), com idade de 60 a 88 anos $(\bar{X}=69,37$ anos; $\mathrm{DP}=5,73)$.

\section{Instrumento}

$O$ instrumento utilizado foi o RESISFOR da bateria AAHPERD. O protocolo de execução do teste foi realizado da seguinte forma: o participante sentado numa cadeira sem braços, com a mão dominante segurando o halter, deveria realizar o maior número de flexões de cotovelo no tempo de 30 segundos, sendo concedidas duas tentativas com intervalo de descanso de um minuto e era registrado o melhor resultado.

Este teste foi realizado duas vezes com halteres de pesos diferentes para cada sexo. Portanto, além do RESISFOR da AAHPERD, que utiliza um halter pesando 1,8 kg para mulheres e 3,6 kg para homens, utilizou-se também o RESISFOR "ajustado", com halteres de $2 \mathrm{~kg}$ e $4 \mathrm{~kg}$ para mulheres e homens, respectivamente. Ambos os testes seguiram o mesmo protocolo de execução, já mencionado. 


\section{Coleta de dados}

O período de aplicação dos testes foi março e dezembro de 2005, na UFSC e dezembro/2005, no GETI/UDESC, ou seja, duas avaliações foram realizadas na UFSC enquanto a UDESC realizou apenas uma. Os dados foram coletados por uma equipe treinada, composta por acadêmicos dos cursos de Educação Física da UFSC e UDESC. A coleta de dados foi realizada nas dependências físicas de ambas as instituições, sendo utilizados procedimentos divergentes somente quanto à ordem de execução do RESISFOR e RESISFOR "ajustado".

A UFSC utilizou os seguintes procedimentos: após as avaliações antropométricas, o indivíduo deslocou-se para outra sala e realizou inicialmente o RESISFOR com o peso proposto pela bateria. Posteriormente, os demais testes motores foram realizados (flexibilidade, equilíbrio, coordenação) e ao final destes, realizou-se o RESISFOR "ajustado". Na UDESC, utilizou-se ordem contrária à citada anteriormente, ou seja, após as avaliações antropométricas, o indivíduo realizou o RESISFOR "ajustado", demais testes motores e por último realizou o RESISFOR.

Salienta-se que, para melhor compreensão dos procedimentos adotados para a realização desse estudo, foram descritos detalhes da avaliação da capacidade funcional que é realizada na UFSC e UDESC, no início e fim de cada ano. Nos últimos cinco anos, ambos os programas utilizam como base, a bateria AAHPERD ${ }^{6,9}$, além da aferição de medidas antropométricas (massa corporal, estatura, circunferências) e demais testes.

\section{Análise Estatística}

Foram realizadas análises descritivas para faixa etária, sexo, local e modalidades praticadas. Para comparar as médias do teste de resistência entre o sexo e entre o local de aplicação, utilizou-se o Teste "t"de student para amostras independentes e Teste "t" pareado, respectivamente. Para associação entre média dos testes de resistência em cada período e em cada faixa etária, foi utilizada ANOVA com post-hoc de Tukey para verificar as respectivas diferenças entre os grupos. O nível de significância adotado foi de $p \leq 0,05$ em todas as análises.

\section{RESULTADOS}

Ao classificar por estrato etário, verificou-se que $33,9 \%$ dos sujeitos estavam classificados na faixa de $65-69$ anos, seguida da faixa de $70-74$ anos $(25,6 \%)$. De acordo com as modalidades de exercício físico, verificou-se que a ginástica é a modalidade mais praticada (tabela 1).

Observou-se que, na comparação das médias obtidas pelos idosos nos testes RESISFOR e RESISFOR "ajustado", a adaptação das cargas do teste não gerou diferenças significativas $(p>0,05)$ para o sexo masculino. Entretanto, para o sexo feminino detectou-se diferença estatisticamente significativa entre os testes na avaliação de dezembro. Detectou-se, ainda, diferença significativa nos testes entre os locais de realização. Nestas diferenças, foram observadas médias significativamente maiores no RESISFOR "ajustado" em detrimento do RESISFOR, ou seja, apesar do primeiro utilizar uma carga pouco maior, verificou-se que o teste ajustado não subestimou o desempenho dos idosos, como pode ser observado na Tabela 2. Em relação ao sexo e local de aplicação do teste, observaram-se diferenças estatisticamente significativas para o sexo feminino e para as médias gerais de dezembro no RESISFOR "ajustado", respectivamente. Salienta-se que não foi realizada comparação entre local, em março, devido a UDESC ter realizado somente uma avaliação (Tabela 2).

Em relação à faixa etária, verificou-se pela ANOVA que existe diferença estatística significativa $(p<0,05)$ entre as médias de flexões de cotovelo realizadas pelos idosos da UFSC, tanto na avaliação de março quanto em dezembro. Ao verificar as diferenças entre os grupos, observou-se que as mesmas foram detectadas entre as faixas etárias mais jovens (60-64 e 65-69 anos) com a faixa etária mais velha (80 anos ou mais), conforme pode ser observado na Tabela 3.

\section{DISCUSSÃO}

A originalidade deste estudo baseia-se na tentativa de facilitar a utilização de um teste (válido e confiável), que avalia a resistência de força de membros superiores em idosos, de uma forma mais prática e acessível. Tal

Tabela 2. Comparação sexo e local, média e desvio padrão (DP) dos testes de resistência de força para membros superiores $(n=407)$.

\begin{tabular}{|c|c|c|c|c|c|c|}
\hline & \multicolumn{3}{|c|}{ Março } & \multicolumn{3}{|c|}{ Dezembro } \\
\hline & \multirow{2}{*}{$\begin{array}{l}\text { RESISFOR } \\
\text { Média(DP) }\end{array}$} & \multicolumn{2}{|c|}{ RESISFOR “ajustado" } & \multirow{2}{*}{$\begin{array}{l}\text { RESISFOR } \\
\text { Média (DP) }\end{array}$} & \multicolumn{2}{|c|}{ RESISFOR "ajustado" } \\
\hline & & Média (DP) & $p$-valor** & & Média (DP) & $p$-valor** \\
\hline Masculino & $20,87(3,90)$ & $21,00(4,55)$ & 0,784 & $20,07(4,76)$ & $20,24(5,19)$ & 0,657 \\
\hline Feminino & $20,65(3,44)$ & $20,64(3,69)$ & 0,919 & $20,20(4,13)$ & $21,54(4,07)$ & $<0,001$ \\
\hline p-valor* & 0,404 & 0,085 & & 0,055 & 0,014 & \\
\hline \multicolumn{7}{|l|}{ Local } \\
\hline UFSC & $20,69(3,51)$ & $20,69(3,82)$ & 0,985 & $21,11(3,74)$ & $22,17(3,76)$ & $<0,001$ \\
\hline UDESC & - & - & - & $16,89(4,20)$ & $18,45(4,69)$ & $<0,001$ \\
\hline p-valor* & - & - & - & 0,214 & 0,05 & \\
\hline
\end{tabular}

- não realizado . * p-valor usando o teste t de Student para amostras independentes. ${ }^{* *} p$-valor usando o teste t pareado 
Tabela 3. Comparação entre a faixa etária e a média do teste de resistência de força para membros superiores $(n=407)$.

\begin{tabular}{|c|c|c|c|c|c|c|c|c|c|c|c|c|}
\hline & \multirow{2}{*}{\multicolumn{4}{|c|}{$\begin{array}{c}\text { MARÇO } \\
\text { UFSC }\end{array}$}} & \multicolumn{8}{|c|}{ DEZEMBRO } \\
\hline & & & & & \multicolumn{4}{|c|}{ UDESC } & \multicolumn{4}{|c|}{ UFSC } \\
\hline & \multicolumn{2}{|c|}{ RESISFOR } & \multicolumn{2}{|c|}{ RESISFOR “ajustado" } & \multicolumn{2}{|c|}{ RESISFOR } & \multicolumn{2}{|c|}{ RESISFOR "ajustado" } & \multicolumn{2}{|c|}{ RESISFOR } & \multicolumn{2}{|c|}{ RESISFOR “ajustado" } \\
\hline & Média & $\mathrm{p}$-valor & Média & $\mathrm{p}$-valor & Média & p-valor & Média & $\mathrm{p}$-valor & Média & $\mathrm{p}$-valor & Média & $\mathrm{p}$-valor \\
\hline F.etária & & 0,002 & & 0,053 & & 0,302 & & 0,200 & & $<0,001$ & & $<0,001$ \\
\hline $60-64$ & $21,47^{\mathrm{A}}$ & & 21,23 & & 17,71 & & 18,81 & & $22,17^{A}$ & & $23,17^{\mathrm{A}}$ & \\
\hline $65-69$ & $21,33^{A}$ & & 21,15 & & 16,46 & & 18,30 & & $21,61^{\mathrm{A}}$ & & $22,75^{\mathrm{A}}$ & \\
\hline $70-74$ & $19,97^{\mathrm{AB}}$ & & 20,52 & & 17,08 & & 18,79 & & $20,80^{A}$ & & $21,67^{A B}$ & \\
\hline $75-79$ & $19,93^{\text {АВ }}$ & & 19,68 & & 17,72 & & 19,81 & & $20,29^{A}$ & & $21,25^{\mathrm{B}}$ & \\
\hline 80 ou> & $18,73^{\mathrm{B}}$ & & 19 & & 14 & & 14,57 & & $17,06^{\mathrm{B}}$ & & $19,06^{\mathrm{B}}$ & \\
\hline
\end{tabular}

Médias seguidas por letras iguais, nas colunas, não diferem pelo Teste de Tukey $(p>0,05)$.

fato mostra-se também uma das limitações encontradas para a discussão do mesmo. Foram encontradas pesquisas abordando o desempenho dos idosos nos testes, mas não especificamente da forma abordado no presente estudo. Assim, a discussão dos dados baseouse nas comparações de desempenho, considerando sexo, faixa etária e local de realização. Outra limitação refere-se ao não controle de algumas variáveis, como: erro inter-avaliador, estado motivacional, dor e patologias que podem ter gerado efeitos sobre o desempenho dos idosos nos testes.

Foram realizadas quatro comparações entre os sexos, sendo duas para os homens (março e dezembro) e duas para as mulheres (março e dezembro). Não foi observada diferença estatisticamente significativa em nenhum dos testes realizados pelos idosos, o que indica que não há diferença entre a utilização de halteres de 3,6 ou $4 \mathrm{~kg}$ pelos homens. Já para as idosas foi encontrada diferença estatística significativa apenas na comparação entre os testes realizados em dezembro. Contudo, mesmo utilizando halteres com um peso maior (2 kg), o RESISFOR “ajustado" apresentou médias superiores ao RESISFOR $(1,8 \mathrm{~kg})$. Este resultado indica que o aumento de 1,8 para $2 \mathrm{~kg}$ não interferiu negativamente no desempenho das idosas no teste e, como não foi observada diferença entre os testes no mês de março, acredita-se que a diferença observada em dezembro possa ser resultado das interferências supra-citadas e não diretamente da carga.

As idosas obtiveram nos testes RESISFOR e RESISFOR "ajustado", em março, uma média de repetições de 20,65 $\pm 3,44$ e 20,64 33,69 , respectivamente e médias de $20,20 \pm 4,13$ e 21,54 44,07 repetições, respectivamente em dezembro. Esses resultados corroboram a pesquisa de Bravo et al..$^{10}$, que observou média de $21,74 \pm 4,89$ repetições. Ao comparar com a média de $29 \pm 6$ repetições, obtida por Zago e Gobi ${ }^{7}$, constatou-se que as médias do presente estudo foram inferiores. Isso pode ser explicado pelas diferenças encontradas entre as idades das duas amostras. No presente estudo, a média de idade das idosas foi de $69,13 \pm 5,59$, enquanto, a média observada por Zago e Gobbi $^{7}$ foi de $64,9 \pm 3,2$ anos.

Com o decorrer da idade, a elasticidade e estabilidade dos músculos, tendões e ligamentos se deterioram, a área transversal dos músculos tornase menor pela atrofia muscular e a massa muscular diminui em proporção ao peso do corpo, o que leva a uma redução da força muscular ${ }^{11}$. Em um estudo de revisão de literatura ${ }^{12}$, os autores verificaram que tanto a força muscular quanto a resistência e a potência sofrem significativa redução com o avançar da idade e que estas reduções podem ser consideradas uns dos principais fatores que interferem na qualidade de vida do idoso. Assim, a otimização da força muscular para os idosos é importante para que estes possam manter-se independentes e autônomos por mais tempo.

No presente estudo, os resultados encontrados na comparação entre as diferentes faixas etárias do grupo amostral corroboram a literatura ${ }^{11,12}$. Verificou-se diferença estatisticamente significativa, principalmente, entre os idosos jovens (60-64 e 65-69 anos) e os idosos velhos (80 anos ou mais), sendo confirmada a tendência das médias a diminuírem com o avançar da idade, em função da perda tanto de força quanto de massa muscular ${ }^{13}$.

Levando em consideração a ordem inversa de execução dos testes nos diferentes locais de aplicação, verificou-se que em ambas as Instituições, as maiores médias encontradas também foram para o RESISFOR "ajustado". A hipótese levantada a respeito da adaptação ao movimento, desgaste fisiológico e local de avaliação não pode ser levado em consideração para explicar tal fato. Observou-se que tanto na UFSC, que realizou o RESISFOR "ajustado" por último, quanto a UDESC, que o realizou primeiro, apresentaram médias superiores estatisticamente significativas em relação à outra execução.

Constatou-se diferença estatística significativa entre as médias das duas Instituições no RESISFOR "ajustado", porém, não foram observadas no RESISFOR. Todavia, esta diferença pode ser explicada pelas atividades principais de cada Instituição, sendo que o programa da UFSC tem como atividade mais praticada a ginástica, enquanto o programa da UDESC, a hidroginástica. A literatura ${ }^{14,15}$ apresenta indícios que ambas as modalidades podem possibilitar ganhos de força para os idosos. Contudo, exercícios resistidos nos quais os indivíduos estejam sujeitos à ação da gravidade provêm melhores resultados para a função 
muscular, uma vez que possibilitam ganhos na força, potência e flexibilidade muscular, além de promover benefícios para o equilíbrio do praticante ${ }^{16}$.

A avaliação da força e da função muscular assume importância no processo de envelhecimento, uma vez que são fundamentais para a autonomia do idoso, devido à grande quantidade de atividades cotidianas, nas quais se necessita de certos níveis de força para executá-las ${ }^{17}$. Logo, a manutenção de bons níveis de força é necessária para o desempenho satisfatório nas tarefas diárias ${ }^{18}$, sejam estas atividades profissionais ou cotidianas ${ }^{19}$ e para a manutenção de uma boa qualidade de vida ${ }^{20}$.

\section{CONCLUSÃO}

Conclui-se com este estudo, que os halteres com pesos ajustados ( $2 \mathrm{~kg}$ para mulheres e $4 \mathrm{~kg}$ para homens) propostos para realizar o RESISFOR podem ser utilizados, visto que, tal adaptação parece não afetar negativamente o desempenho dos idosos.

Diante disso, busca-se facilitar ainda mais a avaliação da capacidade funcional da população idosa, sendo recomendada, para o RESISFOR, a utilização de halteres com peso de $2 \mathrm{~kg}$ para mulheres e $4 \mathrm{~kg}$ para homens, que são de baixo custo, fácil aquisição e acesso no comércio brasileiro.

\section{REFERÊNCIAS BIBLIOGRÁFICAS}

1. World Health Organization. Social development and ageing: crisis or opportunity? Special panel at Geneva 2000. Disponível em: <http://www.who.int/ageing/ publications/development/en/index.html> [2007 mai 08].

2. Instituto Brasileiro de Geografia e Estatística. Indicadores Sociodemográficos - Prospectivos para o Brasil 1991-2030. Projeto UNFPA/BRASIL (BRA/02/ P02). 2006. Disponível em: <http://www.ibge.gov.br/ home/estatistica/populacao/projecao_da_populacao/ publicacao_UNFPA.pdf> [2007 abr 20].

3. Instituto Brasileiro de Geografia e Estatística. Censo demográfico 2000. Rio de Janeiro: Instituto Brasileiro de Geografia e Estatística; 2000.

4. Paixão Jr. CM, Reichenhein ME. Uma revisão sobre instrumentos de avaliação do estado funcional do idoso. Cad Saúde Pública 2005;21(1):07-19.

5. Gobbi S, Gobbi LTB, Ferreira L, Sebastião E. Avaliação física e funcional do idoso. Rev Bras Cineantropom Desempenho Hum 2007;9(S.1):S23-S25.

6. Osness WH, Adrian M, Clark B, Hoeger W, Raab D, Wiswell R. Functional Fitness Assessment for Adults
Over 60 Years. The American Alliance For Health, Physical Education, Recreation and Dance. Association for research, administration, professional councils, and societies. Council on aging and adult development.1900. Association Drive. Reston; 1990.

7. Zago A, Gobbi S. Valores normativos da aptidão funcional de mulheres de 60 a 70 anos. Rev Bras Ciên Mov 2003;11(2):77-86.

8. Benedetti TRB, Mazo GZ, Gobbi S, Lopes MA, Gobbi LTB, Ferreira L, et al. Valores normativos de aptidão funcional em mulheres de 70 a 79 anos. Rev Bras Cineantropom Desempenho Hum 2007;9(1):28-36.

9. Clark BA. Tests for fitness in older adults: AAHPERD Fitness Task Force. JOPERD 1989;60(3):66-71.

10. Bravo G, Pierre Gauthier P, Roy PM, Tessier D, Gaulin $P$, Dubois MF, et al. The functional fitness assessment battery: Reliability and validity data for elderly women. J Aging Phys Act 1994;2(1):67-79.

11. Okuma SS. O idoso e a atividade física. Campinas: Papirus,1998.

12. Lacourt MX, Marini LL. Decréscimo de função muscular decorrente do envelhecimento e a influência na qualidade de vida do idoso: uma revisão de literatura. Rev Bras Ciên Envelhecimento Hum 2006;3(1)114-121.

13. Carvalho J, Soares JMC. Envelhecimento e Força Muscular - breve revisão. Rev Port Ciên Desp 2004;4(3):79-93.

14. Poyhonen T, Sipila S, Keskinen KL, Hautala A, Savolainen J, Malkia E. Effects of aquatic resistance training on neuromuscular performance in healthy women. Med Sci Sports Exerc 2002;34(12):21032109.

15. Carvalho J, Oliveira J, Magalhães J, Ascensão A, Mota J, Soares JMC. Força muscular em idosos II - efeito de um programa complementar de treino na força muscular de idosos de ambos os sexos. Rev Port Ciên Desp 2004;4(1):58-65.

16. Dias RMR, Gurjão ALD, Marucci MFN. Benefícios do treinamento com pesos para a aptidão física de idosos. Acta Fisiatr 2006;13(2):90-95.

17. Monteiro WD, Amorim PRS, Farjalla F, Farinatti PTV. Força muscular e características morfológicas de mulheres idosas praticantes de um programa de atividades físicas. Rev Bras Ativ Fis Saúde 1999;4(1):20-28.

18. Carvalho RBC. Perfil de Aptidão Física Relacionada à Saúde de Pessoas a Partir de 50 Anos Praticantes de Atividades Físicas. [Dissertação de Mestrado-Programa de Pós-Graduação em Educação Física]. Campinas(SP): Universidade Estadual de Campinas;2003.

19. Rikli RE, Jones CJ. Development and validation of functional fitness test for community-residing older adults. J Aging Phys Activity 1999;7(2);129-181.

20. Harries JM, Bassey EJ. Torque- velocity relationship for the knee stensors en women in their 3th na $7 \mathrm{rd}$ decades. Eur J Appl Physio 1990;60;87-90.

\section{Endereço para correspondência}

\section{Lucélia Justino Borges}

Núcleo de Pesquisa em Cineantropometria \& Desempenho Humano

Campus Universitário - Trindade - Caixa Postal: 476

CEP 88010-970 - Florianópolis - SC. Brasil

E-mail: luceliajb@yahoo.com.br
Recebido em 19/08/07

Revisado em 30/11/07

Aprovado em 11/02/08 